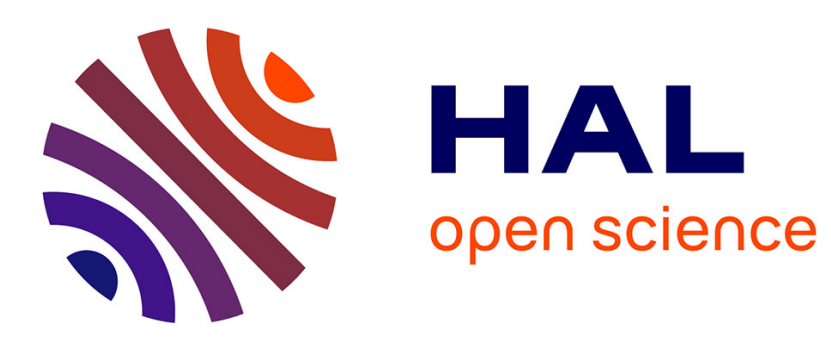

\title{
Viscous stabilizations for high order approximations of Saint-Venant and Boussinesq flows
}

Richard Pasquetti

\section{To cite this version:}

Richard Pasquetti. Viscous stabilizations for high order approximations of Saint-Venant and Boussinesq flows. Marco Bittencourt; Ney Dumont; Jan S. Hesthaven. Lecture Notes in computational Science and Engineering, 119, Springer, pp.519-531, 2017, Spectral and High Order Methods for Partial Differential Equations ICOSAHOM 2016, 978-3-319-65870-4. 10.1007/978-3-319-65870-4_37. hal-01657795

\section{HAL Id: hal-01657795 \\ https://hal.univ-cotedazur.fr/hal-01657795}

Submitted on 21 Dec 2017

HAL is a multi-disciplinary open access archive for the deposit and dissemination of scientific research documents, whether they are published or not. The documents may come from teaching and research institutions in France or abroad, or from public or private research centers.
L'archive ouverte pluridisciplinaire $\mathbf{H A L}$, est destinée au dépôt et à la diffusion de documents scientifiques de niveau recherche, publiés ou non, émanant des établissements d'enseignement et de recherche français ou étrangers, des laboratoires publics ou privés. 


\title{
Viscous stabilizations for high order approximations of Saint-Venant and Boussinesq flows
}

\author{
Richard Pasquetti
}

\begin{abstract}
Two viscous stabilization methods, namely the spectral vanishing viscosity (SVV) technique and the entropy viscosity method (EVM), are applied to flows of interest in geophysics. First, following a study restricted to one space dimension, the spectral element approximation of the shallow water equations is stabilized using the EVM. Our recent advances are here carefully described. Second, the SVV technique is used for the large-eddy simulation of the spatial and temporal development of the turbulent wake of a sphere in a stratified fluid. We conclude with a parallel between these two stabilization techniques.
\end{abstract}

\section{Introduction}

Simulations of flows that can develop stiff gradients generally suffer from numerical instabilities if nothing is done to stabilize the computation. Here we address shallow water flows and the turbulent wake of a sphere in a thermally stratified fluid. Shallow water flows are approximately governed by the Saint-Venant equations, i.e. by a non linear hyperbolic system that can yield shocks depending on the initial conditions. Moreover, one generally expects the numerical scheme to be well balanced and that it can support the presence of dry zones. A large literature is devoted to this questions, see e.g. [1, 24] and references herein. Our simulation of the stratified turbulent wake of a sphere relies on the incompressible Navier-Stokes equations coupled, within the Boussinesq approximation, to an advection-diffusion equation for the temperature. No shocks are in this case expected, but because the smallest scales of the flow cannot be captured by the mesh, here also a stabilization is required. Such a problem is generally addressed using the large-eddy simulation (LES) methodology, see e.g. [20]. In both cases, in the frame of high order methods,

Richard Pasquetti

Université Côte d'Azur, CNRS, Inria, LJAD, France, e-mail: richard.pasquetti@unice.fr. Lab. J.A. Dieudonné (CASTOR project), Parc Valrose, 06108 Nice Cedex 2. 
typically spectrally accurate methods, standard approaches are generally not useful, because implying an unacceptable loss of accuracy.

This paper describes two stabilization techniques that allow, at least formally, to preserve the accuracy of high order methods by introducing relevant viscous terms: The entropy viscosity method (EVM) and the spectral vanishing viscosity (SVV) technique. In Section 2 we develop a spectral element method (SEM) for the SaintVenant system and we stabilize it using the EVM. In Section 3 we use the SVV technique to carry out a Fourier-Chebyshev large-eddy simulation of the turbulent stratified wake of a sphere, on the basis of stabilized Boussinesq equations. To conclude, we provide in Section 4 a parallel between these two stabilization techniques.

\section{Entropy viscosity stabilized approximation of the Saint-Venant system}

This part follows a previous paper [19] where the one-dimensional (1D) SaintVenant system was considered and where we especially focused on problems involving dry-wet transitions. Here this work is extended to the 2D case. Moreover, the treatment of dry zones is improved and the well balanced feature of the scheme is focused on. Comparisons are done with an analytical solution involving dry-wet transitions and the result of a problem combining shocks with dry zones is presented.

The Saint-Venant system results from an approximation of the incompressible Euler equations which assumes that the pressure is hydrostatic and that the perturbations of the free surface are small compared to the water height. Then, from the mass and momentum conservation laws and with $\Omega \subset \mathbb{R}^{2}$ for the computational domain, one obtains equations that describe the evolution of the height $h: \Omega \rightarrow \mathbb{R}^{+}$ and of the horizontal velocity $\boldsymbol{u}: \Omega \rightarrow \mathbb{R}^{2}$ : For $(\boldsymbol{x}, t) \in \Omega \times \mathbb{R}^{+}$:

$$
\begin{aligned}
& \partial_{t} h+\nabla \cdot(h \boldsymbol{u})=0 \\
& \partial_{t}(h \boldsymbol{u})+\nabla \cdot\left(h \boldsymbol{u} \boldsymbol{u}+g h^{2} \mathbb{I} / 2\right)+g h \nabla z=0
\end{aligned}
$$

with $\mathbb{I}$, identity tensor, $\boldsymbol{u} \boldsymbol{u} \equiv \boldsymbol{u} \otimes \boldsymbol{u}, g$, gravity acceleration, and where $z(\boldsymbol{x})$ describes the topography, assumed such that $\nabla z \ll 1$. Let us recall the following properties:

- The system is nonlinear and hyperbolic, which means that discontinuities may develop;

- Assuming that the inlet flow-rate equals the outlet flow-rate, the total mass is preserved;

- The height $h$ is non-negative;

- Rest solutions are stable;

- There exists a convex entropy (actually the energy $E$ ) such that

$$
\partial_{t} E+\nabla \cdot\left(\left(E+g h^{2} / 2\right) \boldsymbol{u}\right) \leq 0, \quad E=h \boldsymbol{u}^{2} / 2+g h^{2} / 2+g h z .
$$


Set $\boldsymbol{q}=h \boldsymbol{u}$ and let $h_{N}(t)\left(\right.$ resp. $\left.\boldsymbol{q}_{N}(t)\right)$ to be the piecewise polynomial continuous approximation of degree $N$ of $h(t)$ (resp. $\boldsymbol{q}(t)$ ). The proposed stabilized SEM relies on the Galerkin approximation of the Saint-Venant system completed with mass and momentum viscous terms. For any $w_{N}, \boldsymbol{w}_{N}$ (scalar and vector valued functions, respectively) spanning the same approximation spaces, in semi-discrete form:

$$
\begin{aligned}
& \left(\partial_{t} h_{N}+\nabla \cdot \boldsymbol{q}_{N}, w_{N}\right)_{N}=-\left(v_{h} \nabla h_{N}, \nabla w_{N}\right)_{N} \\
& \left(\partial_{t} \boldsymbol{q}_{N}+\nabla \cdot I_{N}\left(\boldsymbol{q}_{N} \boldsymbol{q}_{N} / h_{N}\right)+g h_{N} \nabla\left(h_{N}+z_{N}\right), \boldsymbol{w}_{N}\right)_{N}=-\left(v_{q} \nabla \boldsymbol{q}_{N}, \nabla \boldsymbol{w}_{N}\right)_{N}
\end{aligned}
$$

where $v_{h} \propto v_{q}=v$, with $v$ : entropy viscosity (in the rest of the paper we simply use $v_{h}=v_{q}$ ). The usual SEM approach is used here: $I_{N}$ is the piecewise polynomial interpolation operator, based for each element on the tensorial product of GaussLobatto-Legendre (GLL) points, and $(., .)_{N}$ stands for the SEM approximation of the $L^{2}(\Omega)$ inner product, using for each element the GLL quadrature formula which is exact for polynomials of degree less than $2 N-1$ in each variable. The following remarks may be expressed:

- Mass conservation is ensured by the present SEM approximation: Set $w_{N}=1$ in the equation (4); If $\int_{\Gamma} \boldsymbol{q}_{N} \cdot d \Gamma=0$, where $\Gamma$ is the boundary of $\Omega$, then the equalities

$$
\int_{\Omega}\left(\partial_{t} h_{N}+\nabla \cdot \boldsymbol{q}_{N}\right) d \Omega=\int_{\Omega} \partial_{t} h_{N} d \Omega+0=d_{t} \int_{\Omega} h_{N} d \Omega=0
$$

still hold after the SEM discretization. Note however that it is assumed that the Jacobian determinants of the mappings from the reference element $(-1,1)^{2}$ to the mesh elements are piecewise polynomials of degree less than $N$.

- On the contrary, the expected conservation of energy for smooth problems is approximate; This results from the presence of nonlinear terms in (3).

- A stabilization term appears in the mass equation (4). This is required when a high order approximation is involved, i.e. when the scheme numerical diffusion becomes negligible.

- In the momentum equation (5) we do not use the viscous term $\nabla \cdot(h v \nabla \boldsymbol{u})$, which turned out to be less efficient. Indeed, for stabilization purposes the physically relevant stabilization may not be the best suited, see e.g. [8] for the Euler equations.

- Thanks to using $\nabla \cdot I_{N}\left(g h_{N}^{2} \mathbb{I} / 2\right) \approx g h_{N} \nabla h_{N}$ (while $h_{N}^{2}$ is generally piecewise polynomial of degree greater than $N$ ), and thus grouping in (5) the pressure and topography terms, a well balanced scheme is obtained by construction: If $\boldsymbol{q}_{N} \equiv 0$ and $h_{N} \neq 0$, then $h_{N}+z_{N}=$ Constant.

- Another difficulty comes from the positivity of $h_{N}$. This point is addressed at the end of the present Section.

It remains to define the entropy viscosity $v$. To this end we make use of an entropy that does not depend on $z$ but on $\nabla z$, which is of interest, at the discrete level, to get free of the choice of the coordinate system. Taking into account the mass conservation equation (into the entropy equation) one obtains: 


$$
\partial_{t} \tilde{E}+\nabla \cdot\left(\left(\tilde{E}+g h^{2} / 2\right) \boldsymbol{u}\right)+g h \boldsymbol{u} \cdot \nabla z \leq 0, \quad \tilde{E}=h \boldsymbol{u}^{2} / 2+g h^{2} / 2 .
$$

At each time-step, we then compute the entropy viscosity $\boldsymbol{v}(\boldsymbol{x})$ at the GLL grid points, using the following three steps procedure:

- Assuming all variables given at time $t_{n}$, compute the entropy residual, using a backward difference formula, e.g. the BDF2 scheme, to approximate $\partial_{t} \tilde{E}_{N}$

$$
r_{E}=\partial_{t} \tilde{E}_{N}+\nabla \cdot I_{N}\left(\left(\tilde{E}_{N}+g h_{N}^{2} / 2\right) \boldsymbol{q}_{N} / h_{N}\right)+g \boldsymbol{q}_{N} \cdot \nabla z_{N}
$$

where $\tilde{E}_{N}=\boldsymbol{q}_{N}^{2} /\left(2 h_{N}\right)+g h_{N}^{2} / 2$. Then set up a viscosity $v_{E}$ such that:

$$
v_{E}=\beta\left|r_{E}\right| \delta x^{2} / \Delta E,
$$

where $\Delta E$ is a reference entropy, $\beta$ a user defined control parameter and $\delta x$ the local GLL grid-size, defined such that $\delta x^{2}$ equals the surface of the quadrilateral cell (of the dual GLL mesh) surrounding the GLL point, and using symmetry assumptions for the points at the edges and vertices of the element.

- Define a viscosity upper bound based on the wave speeds : $\lambda_{ \pm}=u \pm \sqrt{g h}$ :

$$
v_{\max }=\alpha \max _{\Omega}\left(\left|\boldsymbol{q}_{N} / h_{N}\right|+\sqrt{g h_{N}}\right) \delta x
$$

where $\alpha$ is a $O(1)$ user defined parameter (recall that for the advection equation $\alpha=1 / 2$ is well suited).

- Compute the entropy viscosity:

$$
v=\min \left(v_{\max }, v_{E}\right)
$$

and smooth: (i) locally (in each element), e.g. in 1D: $\left(v_{i-1}+2 v_{i}+v_{i+1}\right) / 4 \rightarrow$ $v_{i}$; (ii) globally, by projection onto the space of the $C^{0}$ piecewise polynomials of degree $N$. Note that operation (ii) is cheap because the SEM mass matrix is diagonal.

The positivity of $h$ is difficult to enforce as soon as $N>1$, so that for problems involving dry-wet transitions the present EVM methodology must be completed. The algorithm that we propose is the following: In dry zones, i.e. for any element $Q_{d r y}$ such that at one GLL point $\min h_{N}<h_{\text {thresh }}$, where $h_{\text {thresh }}$ is a user defined threshold value (typically a thousandth of the reference height):

- Modify the entropy viscosity technique, by using in $Q_{d r y}$ the upper bound first order viscosity:

$$
v=v_{\max } \quad \text { in } Q_{d r y}
$$

- In the momentum equation assume that:

$$
h_{N} g \nabla\left(h_{N}+z_{N}\right) \equiv 0 \quad \text { in } \quad Q_{d r y}
$$


- Moreover, notice that the upper bound viscosity $v_{\max }$ is not local but global, and that the entropy scaling $\Delta E$ used in the definition of $v_{E}$ is time independent. This has improved the robustness of the general approach described in [7].

The numerical results presented hereafter have been obtained using the standard SEM for the discretization in space (GLL nodes for interpolations and quadratures) and the usual forth order Runge-Kutta (RK4) scheme for the discretization in time.

To outline the efficiency of the present EVM for stabilization of Saint-Venant flows involving dry-wet transitions, we first consider a problem for which an analytical solution is available: The planar fluid surface oscillations in a paraboloid [23]. The topography is defined by $z(\boldsymbol{x})=D_{0} x^{2} / L^{2}$ and the exact solution writes, with $\left(x_{1}, x_{2}\right)$ for the Cartesian components of $\boldsymbol{x}$ :

$$
\begin{aligned}
& h=\max \left(0,2 \eta \frac{D_{0}}{L}\left(\frac{x_{1}}{L} \cos (\omega t)-\frac{x_{2}}{L} \sin (\omega t)-\frac{\eta}{2 L}\right)+D_{0}-z\right) \\
& \boldsymbol{u}=-\eta \omega(\sin (\omega t), \cos (\omega t))
\end{aligned}
$$

where $\eta$ determines the amplitude of the motion and with $\omega=\sqrt{2 g D_{0}} / L$. Hereafter we use $L=1 \mathrm{~m}, D_{0}=0.1 \mathrm{~m}$ and $\eta=0.5 \mathrm{~m}$. The dry-wet transition being at the intersection of the paraboloid and of the planar surface of the fluid, in the $\left(x_{1}, x_{2}\right)$ plane the rotating motion of a circle is obtained. Moreover, since $\boldsymbol{u}$ does not depend on $\boldsymbol{x}$, all fluid particles have similar circular trajectories.
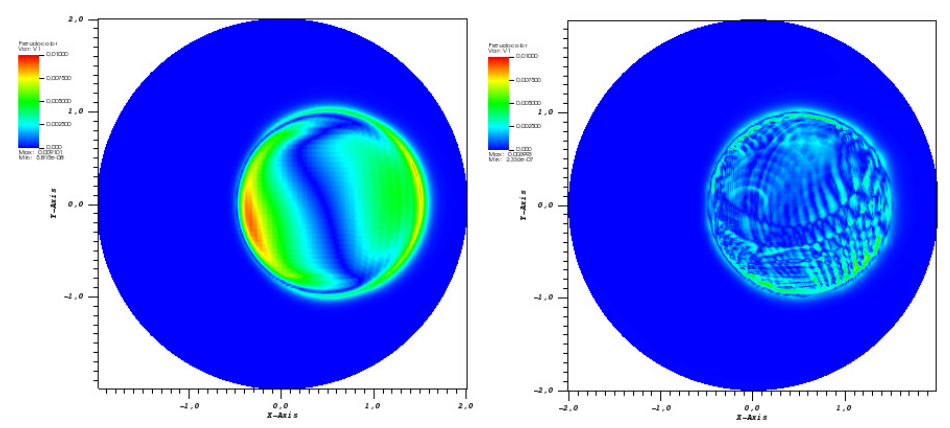

Fig. 1 Planar oscillations in a paraboloid: Error on the height and at the final time for the first order viscosity (left) and EVM (right) solutions (color-scale in $[0,0.01]$ ).

The computational domain is a circle centered at the origin and of diameter 4 . The discretization parameters are the following, number of elements: 2352 , polynomial approximation degree: $N=5$, resulting number of grid-points: 59081, time-step: $2.96 \times 10^{-4}$, EVM control parameters: $\alpha=1, \beta / \Delta E=10$. Moreover, $h_{\text {thresh }}=\max _{\Omega} h_{0} / 500$, with $h_{0} \equiv h(t=0)$, and at the initial time one sets $h_{N}=I_{N}\left(\max \left(h_{0}, h_{\text {thresh }} / 2\right)\right)$. Since here the exact solution is only $C^{0}$ continuous, the computation cannot be spectrally accurate and so the convergence rate would be disappointing. This is why, in order to outline the interest of using a high order 
method like the present stabilized SEM, we prefer to compare the EVM results to results obtained with a first order viscosity. One looks in Fig. 1 at the error $\left|h_{N}-h\right|(\boldsymbol{x})$ at time $t \approx 6 \pi / \omega$, i.e. after three loops of the fluid surface inside the paraboloid. Fig. 1 (left) gives this error field when using the first order viscosity, that is with $v_{h}=v_{q}=v_{\max }$, whereas Fig. 1 (right) shows the result obtained when using the EVM. Clearly, the naive approach that would consist of simply using a first order viscosity for stabilization yields a result by far worse than the one obtained with the EVM. Animations of these error fields clearly confirm such a conclusion. Another test case provided in [23] has also been investigated, namely the axisymmetric oscillations in a paraboloid, and the conclusion is quite similar.
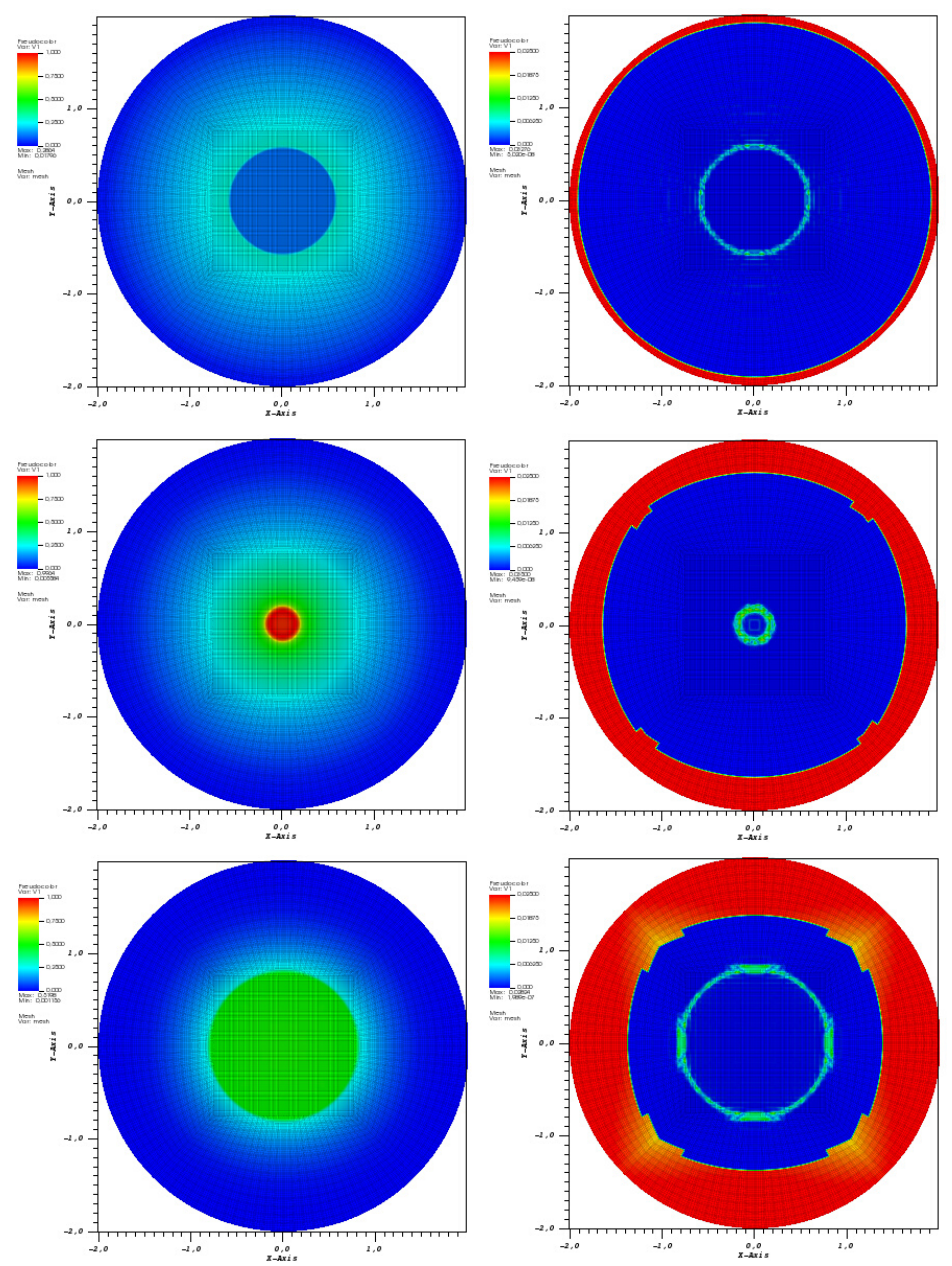

Fig. 2 Axisymmetric oscillations with shocks in a paraboloid: Height (left, in $[0,1]$ ) and entropy viscosity (right, in $[0,0.025]$ ), at $t \approx 1.4$ (top), $t \approx 1.65$ (middle) and $t \approx 1.9$ (bottom). 
To conclude this Section we give the results of a simulation that shows axisymmetric oscillations in a paraboloid and that involves both dry-wet transitions and shocks. The initial condition is the following:

$$
h=\max \left(1-\boldsymbol{x}^{2}, 0\right), \quad \boldsymbol{u}=(0,0),
$$

and at the boundary an impermeability condition together with an homogeneous Neumann condition for $h$ are imposed. The geometry and the space discretization are those used in the first example. Calculations have been made till time $t=5$ with time-step $10^{-4}$, and the EVM control parameters are: $\alpha=1, \beta / \Delta E=20$. As previously, $h_{\text {thresh }}=\max _{\Omega} h_{0} / 500$ and at the initial time $h_{N}=I_{N}\left(\max \left(h_{0}, h_{\text {thresh }} / 2\right)\right)$. Such a flow is alternatively expanding and then retracting towards the paraboloid axis. Fig. 2 shows the flow at three different times, during the first retractionexpansion phase: At $t \approx 1.4$ the velocity field is inwards, at $t \approx 1.65$ it is close to reversal and at $t \approx 1.9$ it is outwards. The height $h_{N}$ (at left) and the entropy viscosity $v$ (at right) are visualized. As desired, the entropy viscosity saturates in dry zones and also focuses at the shock.

\section{Spectral vanishing viscosity for large-eddy simulation of the stratified wake of a sphere}

To demonstrate the interest of using the SVV technique for the computation of turbulent flows, we consider the turbulent wake of a sphere in a thermally stratified fluid. Here we just focus on the main characteristics of the SVV technique and illustrate its capabilities for this particular geophysical flow. Details concerning the physical study may be found in [18].

The SVV technique was initially developed to solve with spectral methods (Fourier / Legendre expansions) hyperbolic problems (non-linear, scalar, 1D, typically the Burgers equation), while (i) preserving the spectral accuracy and (ii) providing a stable scheme $[13,22]$. Later on, say in the 2000's, the SVV technique turned out to be of interest for stabilization of the Navier-Stokes equations and so for the large-eddy simulation (LES) of turbulent flows, see e.g. [10, 11, 12, 15, 16, 25] and references herein.

The basic idea of the SVV stabilization technique is to add some artificial viscosity on the highest frequencies, i.e., to complete the conservation law of some given quantity $u$, assumed to be scalar for the sake of simplicity, with the SVV term :

$$
V_{N} \equiv \varepsilon_{N} \nabla \cdot\left(Q_{N}\left(\nabla u_{N}\right)\right)
$$

where $N$ is again the polynomial degree, $u_{N}$ the numerical approximation of $u, \varepsilon_{N}$ a $O(1 / N)$ coefficient and $Q_{N}$ the so-called spectral viscosity operator, defined to select the highest frequencies: If $Q_{N}$ is omitted in the definition of $V_{N}$, then one recovers the regular diffusion operator. 
In spectral space (Fourier, Chebyshev, Legendre, or any other hierarchical basis) the operator $Q_{N}$ is defined by a set of coefficients $\widehat{Q}_{k}, 0 \leq k \leq N$. Thus, in the 1D periodic case and if Fourier expansions are concerned (trigonometric polynomials are involved in this case):

$$
\left(\widehat{V}_{N}\right)_{k}=-\varepsilon_{N} \widehat{Q}_{k} k^{2}\left(\widehat{u}_{N}\right)_{k}, \quad\left(\widehat{V}_{N}\right)_{-k}={\overline{\left(\widehat{V}_{N}\right)_{k}}}
$$

with $(.)_{k}$ for the $k$-Fourier component, $\overline{(.)}$ for complex conjugate, and where the coefficients $\widehat{Q}_{k}$ are such that $\widehat{Q}_{k}=0$ for $k \leq m_{N}$, with $m_{N}$ a threshold value, and $0<\widehat{Q}_{k} \leq 1, \widehat{Q}_{k}$ being monotonically increasing, for $k>m_{N}$. In the seminal paper [22], $\widehat{Q}_{k}$ was simply chosen as a step function, i.e. with $\widehat{Q}_{k}=1$ if $k>m_{N}$, but it quickly appeared of interest to rather use a smooth approximation. In Fig. 3 two SVV kernels are shown, using the smooth approximation proposed in [13] and with $m_{N}=N / 2$ and $m_{N}=N / 4$.

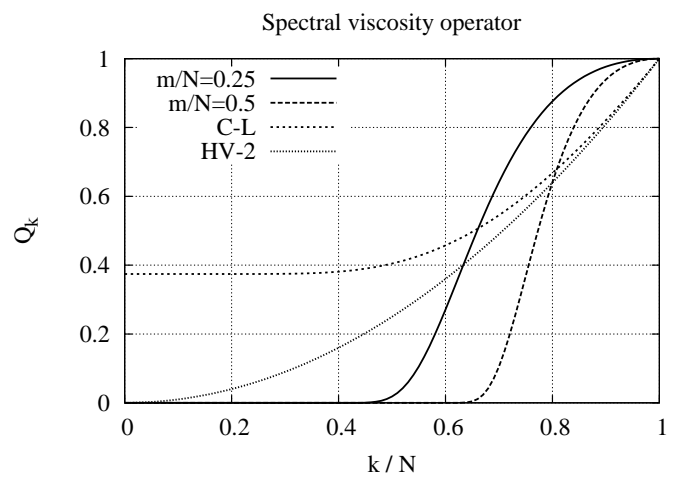

Fig. $3 \widehat{Q}_{k}$ for two SVV kernels, and if using an hyperviscosity (HV) or the Chollet-Lesieur (C-L) subgrid scale model.

One may remark that if defining differently the $\widehat{Q}_{k}$ coefficients, one recovers alternative approaches. Thus, the hyperviscosity stabilization, i.e. such that $V_{N} \propto$ $-\Delta^{2} u_{N}$, so that $\left(\widehat{V}_{N}\right)_{k} \propto k^{4}\left(\widehat{u}_{N}\right)_{k}$, can be recasted in the SVV frame by stating that $\widehat{Q}_{k}=(k / N)^{2}$. In this case, the interpolating curve $\widehat{Q}_{k}(k)$ is simply a parabola, see Fig. 3. Also, if choosing $\varepsilon_{N} \propto N^{-4 / 3}$ and with the curve $\widehat{Q}_{k}(k)$ shown in Fig. 3, one recovers the Chollet-Lesieur spectral viscosity [3], developed for LES in the early 80's and based on the Eddy Damped Quasi-Normal Markovian (EDQNM) theory. This latter approach strongly differs from SVV, since $\widehat{Q}_{k} \neq 0$ even for $k=$ 0 , but both approaches also differ from SVV because the associated kernels nolonger constitute an approximation of a step function. As a result, it is no longer a Laplacean which is acting in the high frequency range. Indeed, if the SVV kernel is simply a step function, then the stabilization term also writes $V_{N}=\varepsilon_{N} \Delta u_{N}^{H}$, where 
$u_{N}^{H}$ is the high frequency part of $u_{N}$. In this perspective, looking at the SVV kernels recently proposed in [14] is also of interest.

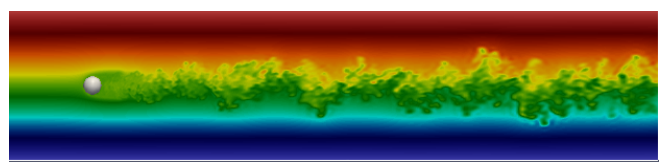

Fig. 4 Temperature field in the median vertical plane at the end of the space development study (color scale: -4 to 4 , from blue to red) [18].

Results obtained with a multi-domain spectral Chebyshev-Fourier solver with SVV stabilization are presented hereafter, see [18] for details. The turbulent wake is generated by a sphere moving horizontally and at constant velocity in a stably stratified fluid, with constant temperature gradient. The flow is assumed governed by the Boussinesq equations and the control parameters are $\operatorname{Pr}=7, \operatorname{Re}=10000, F=25$, for the Prandtl, Reynolds and internal Froude number, respectively. The study is carried out in two steps: First we make a space development study, that is the Galilean frame is associated to the moving sphere; Second, we make a time development study, with a Galilean frame at rest. Such an approach is required to compute the far wake without needing a very elongated computational domain. With respect to anterior works, see e.g. $[4,5]$, the originality of the present study is to make use of the result of the space development study for setting up the initial condition of the temporal development study, thus avoiding the use of a synthetic initial condition.

For the space development study, the computational domain is $(-4.5,30.5) \times$ $(-4,4) \times(-4,4)$. At the initial time, the fluid is stably stratified: $T_{0}=y$, for the dimensionless initial temperature field. The sphere, of unit diameter and centered at $(0,0)$, is modeled by using a volume penalization technique [17]. For the boundary conditions one has: Dirichlet conditions at the inlet, advection at the mean velocity at the outlet, free-slip / adiabaticity conditions on the upper and lower boundaries. The mesh makes use of $12.4 \times 10^{6}$ grid-points. For the velocity components, the SVV parameters are: $m_{N}=N / 2, \varepsilon_{N}=1 / N$, where $N$ is here associated to each of the three axis, whereas for the temperature, $m_{N}=\sqrt{N}, \varepsilon_{N}=1 / N$. The temperature field obtained at the end of the space development study is shown in Fig. 4

For the temporal development study the computational domain is $(-18,18) \times$ $(-4,4) \times(-12,12)$. Note it has been enlarged, since we expect, from the confinement effect due to the stratification, a strong expansion of the wake in the horizontal plane. The initial conditions are set up from the spatial development study, by extraction of the fields obtained at the final time in $(6.5,24.5) \times(-4,4) \times(-4,4)$, see [18] for details. The boundary conditions are: periodicity in streamwise direction, free-slip and adiabaticity elsewhere. The mesh makes use of $27.7 \times 10^{6}$ grid-points. The SVV parameters have not been changed. The flow, at the beginning and at the end of the temporal study, is visualized in Fig. 5. 

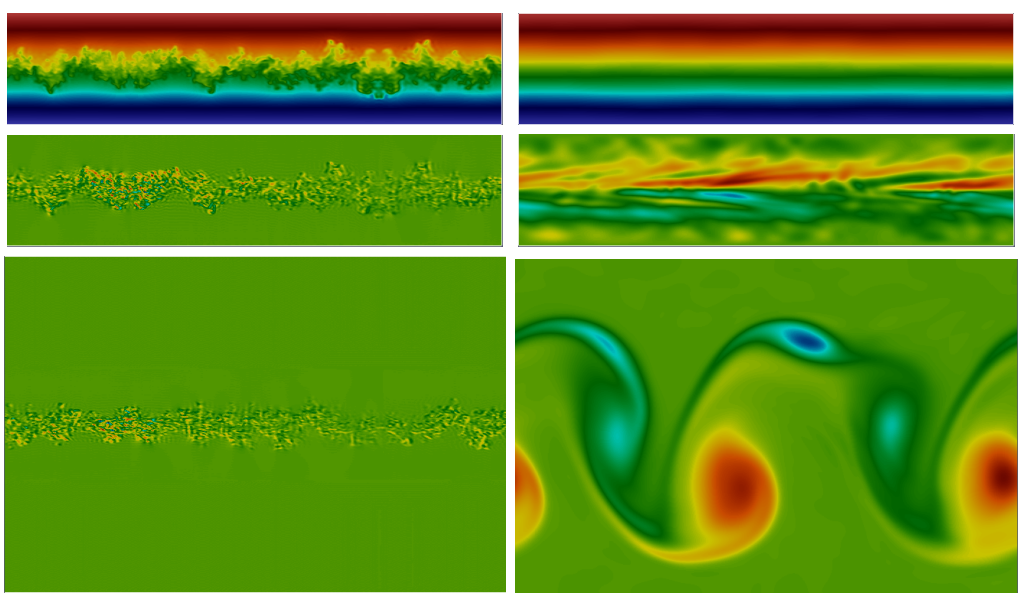

Fig. 5 Temperature and vorticity fields at the beginning (left panel) and at the end (right panel) of the temporal development study. Up: Temperature in the median vertical plane; Middle: Transverse component of the vorticity in the same plane; Bottom: vertical component in the median horizontal plane. The vorticity being much weaker at the final time, the vorticity color scales differ [18].
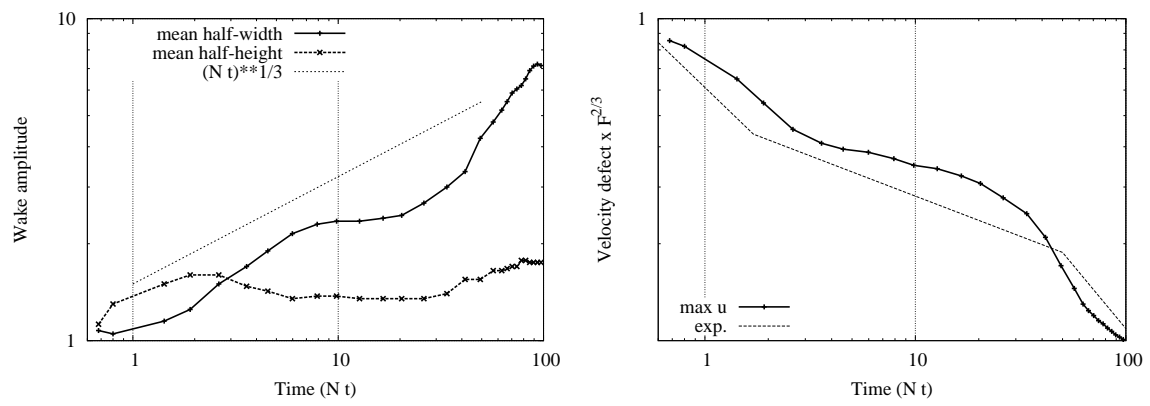

Fig. 6 Wake amplitude (left) and velocity deficit (right) vs time ( $N$, buoyancy frequency) [18].

We conclude with some more quantitative results: In Fig. 6-left one has the evolution of the wake amplitude, both in the vertical and horizontal planes, which clearly points out the confinement effect of the stratification (here $N t$ is a dimensionless time, with $N$ for the Brunt-Väisälä buoyancy angular frequency of the fluid at rest); Fig. 6-right shows the evolution of the velocity defect. This latter curve is in reasonable agreement with the "universal curve" of [21], where three phases in the development of sphere stratified wakes are described: First the 3D phase, then the non equilibrium (NEQ) phase and finally the quasi two 2D (Q2D) phase. Tab. 7 compares the experimental results to the present numerical ones, in terms of characteristic quantities of the velocity defect evolution. 


\begin{tabular}{|c|c|c|c|c|c|}
\hline & $N t_{I}$ & $N t_{I I}$ & 3D rate & NEQ rate & Q2D rate \\
\hline [21] & $1.7 \pm 0.3$ & $50 \pm 15$ & $-2 / 3$ & $-0.25 \pm 0.4$ & $-0.76 \pm 0.12$ \\
SVV-LES & 2.4 & 30 & $\sim-2 / 3$ & -0.2 & $\sim-0.76$ \\
\hline
\end{tabular}

Fig. 7 Critical times $N t_{I}$ and $N t_{I I}$ that correspond to the 3D-NEQ and NEQ-Q2D transitions, respectively, and rates of variation of the velocity defect in each of the three phases.

\section{Concluding parallel between the EVM and SVV stabilizations}

Two viscous stabilizations, namely the entropy viscosity method (EVM) and the spectral vanishing viscosity (SVV) technique, have been successfully implemented in high order approximations of geophysical flows: With EVM shallow water flows involving dry-wet transitions have been addressed and with SVV the turbulent wake of a sphere in a thermally stratified fluid has been investigated. We conclude with a parallel between these two approaches:

- Both SVV and EVM are viscosity methods first developed for hyperbolic problems, see [6, 22].

- EVM is non-linear while SVV is linear. SVV is thus not costly, since its implementation is done in preprocessing step. Moreover, because of this linear feature it is very robust and so well adapted to the LES of turbulent flows.

- Both EVM and SVV preserve the accuracy of the numerical approximation. This is of course essential when high order methods are concerned.

- SVV is not Total Variation Diminishing (TVD) and EVM is not fully TVD, since this depends on the values of the EVM control parameters ( $\alpha$ and $\beta$ ). For SVV, a post processing stage for removing spurious oscillations has been suggested [13].

- A theory exists for SVV [22], whereas no complete theory is available for EVM. Some theoretical results, restricted to some specific time schemes and space approximations, are however available [2].

- EVM may be used with various numerical methods, since based on a physical argument, including the standard finite element method (FEM), finite volume methods etc.. SVV is restricted to spectral type methods, e.g. high order FEMs like the SEM.

- SVV has proved to be of interest for LES (SVV-LES). Preliminary numerical experiments are now available for EVM [9], but additional tests and comparisons remain needed to check if EVM is not too diffusive and robust enough when turbulent flows are concerned.

Acknowledgements Part of this work was made at the Dpt of Mathematics of National Taiwan University in the frame of the Inria project AMOSS. 


\section{References}

1. C. Berthon, F. Marche, A positive preserving high order VFRoe scheme for shallow water equations: a class of relaxation schemes, SIAM J. Sci. Comput., 30, 2587-2612, 2008.

2. A. Bonito, J.L. Guermond, B. Popov, Stability analysis of explicit entropy viscosity methods for non-linear scalar conservation equations, Math. Comp., 83, 1039-1062, 2014.

3. J. P. Chollet, M. Lesieur, Parametrisation of small scales of three-dimensional isotropic turbulence utilizing spectral closures, J. of Atmospheric Sciences, 38, 2747-2757, 1981.

4. P.J. Diamessis, J.A. Domaradzki, J.S. Hesthaven, A spectral multidomain penalty method model for the simulation of high Reynolds number localized incompressible stratified turbulence, J. Comput. Phys., 202, 298-322, 2005.

5. D.G. Dommermuth, J.W. Rottman, G.E. Innis, E.V. Novikov, Numerical simulation of the wake of a towed sphere in a weakly stratified fluid, J. Fluid Mech., 473, 83-101, 2002.

6. J.L. Guermond, R. Pasquetti, Entropy-based nonlinear viscosity for Fourier approximations of conservation laws, C.R. Acad. Sci. Paris, Ser. I, 346, 801-806, 2008.

7. J.L. Guermond, R. Pasquetti, B. Popov, Entropy viscosity method for non-linear conservation laws, J. of Comput. Phys., 230 (11), 4248-4267, 2011.

8. J.L. Guermond, B. Popov, Viscous regularization of the Euler equations and entropy principles, SIAM J. Appl. Math., 74 (2), 284-305, 2014.

9. J.-L. Guermond, A. Larios, T. Thompson, Validation of an entropy-viscosity model for large eddy simulation, Direct and Large Eddy-Eddy Simulation IX, ECOFTAC Series, Vol. 20, 43-48, 2015.

10. G. S. Karamanos, G. E. Karniadakis, A spectral vanishing viscosity method for large-eddy simulation, J. Comput. Phys., 163, 22-50, 2000

11. R. M. Kirby, S. J. Sherwin, Stabilisation of spectral / $h p$ element methods through spectral vanishing viscosity: Application to fluid mechanics, Comput. Methods Appl. Mech. Engrg., 195, 3128-3144, 2006.

12. K. Koal, J. Stiller, H.M. Blackburn, Adapting the spectral vanishing viscosity method for large-eddy simulations in cylindrical configurations, J. of Comput. Phys., 231, 3389-3405, 2012

13. Y. Maday, S. M. O. Kaber, E. Tadmor, Legendre pseudo-spectral viscosity method for nonlinear conservation laws, SIAM J. Numer. Anal., 30, 321-342, 1993.

14. R.C. Moura, S.J. Sherwin, J. Peiró, Eigensolution analysis of spectral/hp continuous Galerkin approximations to advectiondiffusion problems: Insights into spectral vanishing viscosity, J. Comput. Phys., 307, 401-422, 2016.

15. R. Pasquetti, Spectral vanishing viscosity method for large-eddy simulation of turbulent flows, J. Sci. Comp., 27, 365-375, 2006.

16. R. Pasquetti, E. Séverac, E. Serre, P. Bontoux, M. Schäfer, From stratified wakes to rotor-stator flows by an SVV-LES method, Theor. Comput. Fluid Dyn., 22, 261-273, 2008.

17. R. Pasquetti, R. Bwemba, L. Cousin, A pseudo-penalization method for high Reynolds number unsteady flows, Applied Numerical Mathematics, 58 (7), 946-954, 2008.

18. R. Pasquetti, Temporal / spatial simulation of the stratified far wake of a sphere, Computers \& Fluids, 40, 179-187, 2010

19. R. Pasquetti, J.L. Guermond, B. Popov, Stabilized spectral element approximation of the Saint-Venant system using the entropy viscosity technique, in Lecture Notes in computational Science and Engineering : Spectral and High Order Methods for Partial Differential Equations - ICOSAHOM 2014, vol. 106, Springer, 397-404, 2015.

20. P. Sagaut, Large Eddy Simulation for Incompressible Flows, Springer Berlin Heidelberg, 2006.

21. G.R. Spedding, F.K. Browand, A.M. Fincham, The evolution of initially turbulent bluff-body wakes at high internal Froude number, J. Fluid Mech., 337, 283-301, 1997.

22. E. Tadmor, Convergence of spectral methods for nonlinear conservation laws, SIAM J. Numer. Anal., 26, 30-44, 1989.

23. W.C. Thacker, Some exact solutions to the nonlinear shallow-water wave equations, J. Fluid Mech., 107, 499-508, 1981.

24. Y. Xing, X. Zhang, Positivity-preserving well-balanced discontinuous Galerkin methods for the shallow water equations on unstructured triangular meshes, J. Sci. Comput., 57, 19-41, 2013.

25. C.J. Xu, R. Pasquetti, Stabilized spectral element computations of high Reynolds number incompressible flows, J. Comput. Phys., 196, 680-704, 2004. 